\begin{tabular}{|c|l|}
\hline Title & A uniqueness theorem and the Myrberg phenomenon \\
\hline Author(s) & Hay ashi, Mikihiro; Nakai, Mitsuru \\
\hline Citation & Journal d'A nalyse Mathématique, 76(1), 109-136 \\
\hline https:/doi.org/10.1007/BF02786932 & 1998 \\
\hline Issue Date & http://hdl.handle.net/2115/43862 \\
\hline Doc URL & The original publication is available at www.springerlink.com \\
\hline Rights & article (author version) \\
\hline Type & HN9609.pdf \\
\hline File Information & \\
\hline
\end{tabular}

Instructions for use 


\title{
A UNIQUENESS THEOREM AND THE MYRBERG PHENOMENON
}

\author{
MIKIHIRO HAYASHI AND MITSURU NAKAI
}

\begin{abstract}
The Dirichlet irregularity of the origin which is a boundary point of a Zalcman domain $R$ is shown to be sufficient but not necessary for the occurrence of the Myrberg phenomenon $H^{\infty}(\tilde{R})=H^{\infty}(R) \circ \varphi$ for a two sheeted unlimited smooth covering surface $\tilde{R}$ of $R$ with the projection map $\varphi$. The importance of the uniqueness theorem in such a study of the Myrberg phenomenon is stressed. An invalidity condition of the Myrberg phenomenon for the covering surface $(\tilde{R}, R, \varphi)$ is also considerd.
\end{abstract}

\section{Introduction}

1.1. We denote by $H^{\infty}(W)$ the Banach space of bounded holomorphic functions $f$ on a Riemann surface $W$ equipped with the supremum norm $\|f\|_{\infty}$. Let $\tilde{W}$ be an unlimited possibly infinitely branched covering surface of a Riemann surface $W$ with the projection map $\varphi$. We say that the Myrberg phenomenon occurs for the covering surface $(\tilde{W}, W, \varphi)$ if we have

$$
H^{\infty}(\tilde{W})=H^{\infty}(W) \circ \varphi .
$$

We are particulary interested in the case when $\tilde{W}$ is an unlimited two sheeted smooth (i.e. unbranched) covering surface of a bounded plane region $W$ (cf. [5], [6], [7], [8], [4]). In this paper we consider the case $W$ is a certain Zalcman domain described below.

1.2. We denote by $\Delta(c, r)(\bar{\Delta}(c, r)$, resp.) the open (closed, resp.) disc in the complex plane $\mathbb{C}$ with radius $r>0$ centered at $c$. For simplicity we denote by $\Delta$ and $\Delta_{0}$ the unit disc $\Delta(0,1)$ and the punctured unit disc $\Delta(0,1) \backslash\{0\}$, respectively. Let $\left(c_{n}\right)_{n \geq 1}$ be a strictly decreasing sequence with $0<c_{n}<1$ converging to 0 and $\left(r_{n}\right)_{n \geq 1}$ a sequence of positive numbers such that

$$
c_{n+1}+r_{n+1}<c_{n}-r_{n}(n \in \mathbb{N}), c_{1}+r_{1}<1,
$$

where $\mathbb{N}$ is the set of positive integers. The condition (1.2) simply says that discs $\bar{\Delta}\left(c_{n}, r_{n}\right)$ are contained in $\Delta_{0}$ and mutually disjoint. Following [16] we consider the

To complete the present work the first (second, resp.) named author was supported in part by Grant-in-Aid for Scientific Research, No. 07640154 (07640196, 08640194, 09640180, 09640230, resp.), Japanese Ministry of Education, Science and Culture. 
domain

$$
R:=R\left(c_{n}, r_{n}\right):=\Delta_{0} \backslash \bigcup_{n \in \mathbb{N}} \bar{\Delta}\left(c_{n}, r_{n}\right),
$$

which serves as the simplest example of plane regions of infinite connectivity in various problems related to the class of bounded holomorphic functions (cf. e.g. $[2])$. A region $R$ with the form as in (1.3) will be referred to as a Zalcman domain, or an L-domain in the terminology of [16].

1.3. Let $\left(c_{n}\right)_{n \geq 1}$ and $R$ be as in 1.2. Consider an unlimited two sheeted covering surface $\tilde{\Delta}_{0}$ of $\Delta_{0}$ with $\left\{c_{n}: n \in \mathbb{N}\right\}$ as the set of projections of its branch points. The projection map is denoted by $\varphi$. The covering surface $\left(\tilde{\Delta}_{0}, \Delta_{0}, \varphi\right)$ naturally gives rise to a covering surface $(\tilde{R}, R, \varphi)$ which is an unlimited smooth (i.e. unbranched) two sheeted covering surface. In his celebrated paper [10] (see also [14]) Myrberg first pointed out that $(1.1)$ holds for $\left(\tilde{\Delta}_{0}, \Delta_{0}, \varphi\right)$. This is the reason why the name Myrberg is attached to the relation (1.1).

Myrberg's proof goes as follows. Choose an arbitrary $g$ in $H^{\infty}\left(\tilde{\Delta}_{0}\right)$ and we are to show that $g\left(z_{+}\right)=g\left(z_{-}\right)$for every $z$ in $\Delta_{0}$, where $\varphi^{-1}(z)=\left\{z_{+}, z_{-}\right\}$under the convention that $z_{+}=z_{-}$for $z=c_{n}(n \in \mathbb{N})$. Consider the function $f$ in $H^{\infty}\left(\Delta_{0}\right)$ determined by

$$
f(z)=\left(g\left(z_{+}\right)-g\left(z_{-}\right)\right)^{2}
$$

for every $z$ in $\Delta_{0}$. By the Riemann removability theorem $f$ can be continued to $\Delta$ so as to be in $H^{\infty}(\Delta)$. Since $f\left(c_{n}\right)=0(n \in \mathbb{N})$ and $c_{n} \rightarrow 0(n \rightarrow \infty)$, the classical uniqueness theorem implies $f \equiv 0$ and we are done.

Looking at this proof one might feel that the presence of too many branch points $\left\{\varphi^{-1}\left(c_{n}\right): n \in \mathbb{N}\right\}$ is essential in the occurrence of the Myrberg phenomenon for $\left(\tilde{\Delta}_{0}, \Delta_{0}, \varphi\right)$ and that $(1.1)$ is no longer true for the smooth covering surface $(\tilde{R}, R, \varphi)$. For this reason it was a bit surprising when we found (cf. [5]) that the Myrberg phenomenon can occur even for a certain $(\tilde{R}, R, \varphi)$ in spite of the complete lack of branch points. One of the main purpose of this paper is to clarify why such a phenomenon can occur. We will show in $\S 3$ that there is a case where a kind of uniqueness theorem can hold for $H^{\infty}(R)$ at $z=0$ and that, as in the original case of Myrberg, this uniqueness theorem yields the validity of the Myrberg phenomenon for this $(\tilde{R}, R, \varphi)$. The uniqueness theorem for $H^{\infty}(R)$ at $z=0$ we have in mind is the following: if $\lim _{z<0, z \rightarrow 0} f^{(n)}(z)=0$ for every $n=0,1, \ldots$ for an $f$ in $H^{\infty}(R)$, then $f \equiv 0$ on $R$.

1.4. To facilitate our study, hereafter throughout this paper unless the contrary is explicitly stated, we restrict $\left\{c_{n}: n \in \mathbb{N}\right\}$ to the special sequence $c_{n}=2^{-n}(n \in \mathbb{N})$ and we introduce a sequence $(N(n))_{n \geq 1}$ to relate $\left(r_{n}\right)_{n \geq 1}$ with $\left(c_{n}\right)_{n \geq 1}$ by

$$
r_{n}=c_{n}^{N(n)}=2^{-n N(n)}(n \in \mathbb{N}) .
$$

The condition (1.2) in the present case takes the following form:

$$
2^{-n N(n)}+2^{-(n+1) N(n+1)}<2^{-(n+1)}(n \in \mathbb{N}) .
$$

Observe that (1.5) implies $N(n)>1+1 / n(n \in \mathbb{N})$ and conversely $N(n)>1+$ $2 / n(n \in \mathbb{N})$ implies (1.5). The sequence $(N(n))_{n \geq 1}$ with (1.5) will be referred to 
as being admissible. We will thus consider, using the notation $R(N(n))$ in place of $R\left(2^{-n}, 2^{-n N(n)}\right)$ for simplicity, the following special Zalcman domain

$$
R:=R(N(n)):=\Delta_{0} \backslash \bigcup_{n \in \mathbb{N}} \bar{\Delta}\left(2^{-n}, 2^{-n N(n)}\right),
$$

which varies depending upon the choice of admissible sequence $(N(n))_{n \geq 1}$ while the sequence $\left(2^{-n}\right)_{n \geq 1}$ of centers are always fixed.

1.5. We are then interested in finding conditions on $(N(n))_{n \geq 1}$ under which the Myrberg phenomenon occurs or does not occur for the covering surface $(\tilde{R}, R, \varphi)$ with $R=R(N(n))$. We obtained in our former papers ([5], [6]) the following result.

Theorem A. If the point $z=0$ is irregular for the region $R=R(N(n))$ in the sense of potential theory (or equivalently, $(N(n))_{n \geq 1}$ diverges so rapidly as to satisfy $\left.\sum_{n \geq 1} 1 / N(n)<\infty\right)$, then the Myrberg phenomenon occurs for the covering surface $(\tilde{R}, R, \varphi)$.

It is a natural question to ask whether the sufficiency condition (i.e. the irregularity at $z=0$ ) in the above result is also necessary for the validity of the Myrberg phenomenon for $(\tilde{R}, R, \varphi)$ or not. The main purpose of this paper is to answer the question in the negative. This will be achieved as follows. We first prove in $\S 4$ below, as mentioned above in 1.3 , the main theorem that the validity of what we call the uniqueness theorem for $H^{\infty}(R)$ at $z=0$ implies the occurrence of the Myrberg phenomenon for $(\tilde{R}, R, \varphi)$ (Theorem 4.1). Although it is not really needed, we prove in $\S 5$ below that the irregularity of $z=0$ implies the validity of the uniqueness theorem for $H^{\infty}(R)$ at $z=0$ (Proposition 5.1), and by this together with the above main result we obtain an alternate proof of Theorem A above as Theorem 5.1. We then prove in $\S 6$ below that a kind of quasi analyticity condition at $z=0$ for $R$ again implies the uniqueness theorem for $H^{\infty}(R)$ at $z=0$ (Proposition 6.1). Once again using the main theorem 4.1 we thus obtain the new result that what we call the quasi analyticity condition at $z=0$ implies the validity of the Myrberg phenomenon for $(\tilde{R}, R, \varphi)$ (Theorem 6.1). In $\S 7$ below we give an example of an admissible sequence $(N(n))_{n \geq 1}$ giving the regularity of $z=0$ and at the same time the quasi analyticity of $z=0$, which deduces the above negative solution required (Theorem 7.1).

In the final $\S 8$ below we append a study to seek the invalidity condition for the Myrberg phenomenon, which is a negative approach to pursue the complete condition for the occurence of the Myrberg phenomenon. We will show that the slowly increasingness condition

$$
\sup _{n \in \mathbb{N}} n\left(N(n)-\frac{n+1}{2}\right)<\infty
$$

implies the invalidity of the Myrberg phenomenon for $(\tilde{R}, R, \varphi)$ with $R=R(N(n))$ (Theorem 8.1). 


\section{The Cauchy integral formula}

2.1. Let $R=R\left(c_{n}, r_{n}\right)$ be a Zalcman domain in (1.3). For simplicity we denote by $\Delta_{n}$ the closed disc $\bar{\Delta}\left(c_{n}, r_{n}\right)$ and by $\Gamma_{n}$ the circle $\partial \Delta_{n}$ as the set but given negative orientation for $n \in \mathbb{N}$ whereas we denote by $\Delta_{0}$ the punctured open unit disc $\Delta(0,1) \backslash\{0\}$ and we denote by $\Gamma_{0}$ the circle $\partial \Delta(0,1)$ with the usual positive orientation. Then we have

$$
R=\Delta_{0} \backslash \bigcup_{n \in \mathbb{N}} \Delta_{n}
$$

Any function $f$ in $H^{\infty}(R)$ has nontangential boundary values almost everywhere on each $\Gamma_{n}$ defining a function in $L^{\infty}\left(\Gamma_{n}\right)$, which we also denote by $f$ so that $f(\zeta)$ can be considered for almost every $\zeta$ in each $\Gamma_{n}(n=0,1, \ldots)$. Then we have the following Cauchy integral formula for functions $f$ in $H^{\infty}(R)$ (cf. e.g. [16],[2]):

$$
f^{(\ell)}(z)=\sum_{n=0}^{\infty} \frac{\ell !}{2 \pi i} \int_{\Gamma_{n}} \frac{f(\zeta)}{(\zeta-z)^{\ell+1}} d \zeta \quad(z \in R ; \ell=0,1, \ldots),
$$

where $f^{(\ell)}$ is the $\ell^{\text {th }}$ derivative of $f$ on $R$ with the convention $f^{(0)}=f$. It is sometimes convenient to consider $f$ to be contained in $L^{\infty}(\Gamma)$ with $\Gamma=\cup_{n=0}^{\infty} \Gamma_{n}$ and to use the notation

$$
\frac{\ell !}{2 \pi i} \int_{\Gamma} \frac{f(\zeta)}{(\zeta-z)^{\ell+1}} d \zeta
$$

to mean the right hand side of (2.1).

To show the validity of (2.1) we consider the positive number $\delta_{m}$ that is the additive mean of $c_{m+1}+r_{m+1}$ and $c_{m}-r_{m}(m \in \mathbb{N})$ and we denote by $\gamma_{m}$ the circle $|z|=\delta_{m}$ with negative orientation. For each $z$ in $R$ we take a sufficiently large $m$ such that $z \in R_{m}$, the region bounded by $\Gamma_{0}, \Gamma_{1}, \ldots, \Gamma_{m}$ and $\gamma_{m}$. The region

$$
R_{m}^{\varepsilon}:=\left\{w \in R_{m}: \operatorname{dist}\left(w, \partial R_{m}\right)>\varepsilon\right\}
$$

is a relatively compact subregion of $R_{m}$ such that $\partial R_{m}^{\varepsilon}$ consists of $m+2$ mutually disjoint circle concentric to each corresponding component of $\partial R_{m}$ for sufficiently small $\varepsilon>0$. The usual Cauchy integral formula yields

$$
f^{(\ell)}(z)=\frac{\ell !}{2 \pi i} \int_{\partial R_{m}^{\varepsilon}} \frac{f(\zeta)}{(\zeta-z)^{\ell+1}} d \zeta .
$$

Since $f \mid \partial R_{m}^{\varepsilon}$ is uniformly bounded as $\varepsilon \downarrow 0$ and converges to $f \mid \partial R_{m}$ almost everywhere in a suitable parametrization as $\varepsilon \downarrow 0$, the Lebesgue dominated convergence theorem implies

$$
\begin{aligned}
f^{(\ell)}(z) & =\frac{\ell !}{2 \pi i} \int_{\partial R_{m}} \frac{f(\zeta)}{(\zeta-z)^{\ell+1}} d \zeta \\
& =\sum_{0 \leq n \leq m} \frac{\ell !}{2 \pi i} \int_{\Gamma_{n}} \frac{f(\zeta)}{(\zeta-z)^{\ell+1}} d \zeta+\frac{\ell !}{2 \pi i} \int_{\gamma_{m}} \frac{f(\zeta)}{(\zeta-z)^{\ell+1}} d \zeta .
\end{aligned}
$$

The integral over $\gamma_{m}$ is bounded by $\ell !\left(\sup _{R}|f|\right) \delta_{m} /\left(|z|-\delta_{m}\right)^{\ell+1}$ and thus goes to zero as $m \rightarrow \infty$, which yields (2.1). 
2.2. Thus far no restrictions are imposed upon $\left(c_{n}, r_{n}\right)_{n \geq 1}$ except for the condition (1.2), by which the best conclusion we could make is (2.1). We now consider the following condition for $\left(c_{n}, r_{n}\right)_{n \geq 1}$ :

$$
\sum_{n=1}^{\infty} r_{n}\left(c_{n}-r_{n}\right)^{-(\ell+1)}<\infty \quad(\text { for all } \ell=0,1, \ldots) .
$$

Under the condition (2.2) it is easy to see that

$$
\sum_{n=0}^{\infty} \frac{\ell !}{2 \pi i} \int_{\Gamma_{n}} \frac{f(\zeta)}{\zeta^{\ell+1}} d \zeta=\frac{\ell !}{2 \pi i} \int_{\Gamma} \frac{f(\zeta)}{\zeta^{\ell+1}} d \zeta
$$

is well defined, which we denote by $f^{(\ell)}(0)$ :

$$
f^{(\ell)}(0)=\frac{\ell !}{2 \pi i} \int_{\Gamma} \frac{f(\zeta)}{\zeta^{\ell+1}} d \zeta
$$

Clearly the formal $\ell^{\text {th }}$ derivative $f^{(\ell)}$ of $f$ at $z=0$ just defined coincides with the genuine $\ell^{\text {th }}$ derivative of $f$ at $z=0$ if $f$ is holomorphic at $z=0$. It is also important that under the condition (2.2) we have

$$
f^{(\ell)}(0)=\lim _{z<0, z \rightarrow 0} f^{(\ell)}(z) \quad(\ell=0,1, \ldots) .
$$

In fact, observe that $\int_{\Gamma_{n}}|d \zeta|=2 \pi r_{n}$ and $\left|f(\zeta) /(\zeta-z)^{\ell+1}\right|$ is dominated by $\left(\sup _{R}|f|\right) \cdot\left(c_{n}-r_{n}\right)^{-(\ell+1)}$ on $\Gamma_{n}$ for $z$ in the negative real line, and hence $\mid f(\zeta) /(\zeta-$ $z)^{\ell+1} \mid$ is dominated by an integrable step function on $\Gamma$ for every $z<0$. We can thus apply the Lebesgue dominated convergence theorem to deduce (2.4) by making $z \rightarrow 0(z<0)$ in $(2.1)$. (cf. [3], where $(2.4)$ is proved for more general domains.)

2.3. Assuming (2.2) we can associate the following functions $F_{\ell}(z)$ with $f(z)$ given by

$$
F_{\ell}(z):=\frac{1}{2 \pi i} \int_{\Gamma} \frac{f(\zeta)}{\zeta^{\ell}(\zeta-z)} d \zeta \quad(z \in R ; \ell=0,1, \ldots)
$$

Clearly the right hand side of the above is well defined for every $z \in R \cup\{0\}$ and

$$
F_{0}(z) \equiv f(z)(z \in R), \ell ! F_{\ell}(0)=f^{(\ell)}(0)(\ell \in \mathbb{N}) .
$$

By a simple direct calculation using $(2.1)(\ell=0),(2.3)$ and $(2.5)$, we obtain the following identity:

$$
f(z)-\sum_{k=0}^{\ell-1} \frac{f^{(k)}(0)}{k !} z^{k}=z^{\ell} F_{\ell}(z)(\ell=0,1, \ldots) .
$$

If $c_{n}=2^{-n}$ and $r_{n}=2^{-n N(n)}$, we consider the following divergence condition for $(N(n))_{n \geq 1}$ :

$$
\lim _{n \rightarrow \infty} N(n)=\infty
$$


The condition above assures more than sufficiently $(N(n))_{n \geq 1}$ satisfy (1.5) if the first few terms are modified, if necessary. Since

$$
\sum_{n=1}^{\infty} r_{n}\left(c_{n}-r_{n}\right)^{-\ell} \leq \sum_{n=1}^{\infty} 2^{-n N(n)}\left(2^{-n} / 2\right)^{-\ell}=\sum_{n=1}^{\infty} 2^{-n(N(n)-\ell-\ell / n)}
$$

for $\ell=1,2, \ldots$, the condition (2.8) implies (2.2). The following result now follows instantly from (2.7).

Lemma 2.1. Suppose an admissible sequence $(N(n))_{n \geq 1}$ satisfies $(2.8)$. Then the condition $f^{(\ell)}(0)=0(\ell=0,1, \ldots)$ for an $f$ in $H^{\infty}(R)$ holds if and only if

$$
f(z) \equiv z^{\ell} F_{\ell}(z) \quad(z \in R ; \ell=0,1, \ldots) .
$$

\section{The Uniqueness TheOREM}

3.1. In this section 3 we also consider a general Zalcman domain $R\left(c_{n}, r_{n}\right)$ (cf. 1.2) in addition to the special Zalcman domain $R(N(n))$. We say that the uniqueness theorem is valid for $H^{\infty}\left(R\left(c_{n}, r_{n}\right)\right)$ at $z=0$ if the following condition is satisfied: if an $f$ in $H^{\infty}\left(R\left(c_{n}, r_{n}\right)\right)$ satisfies the condition

$$
\lim _{z<0, z \rightarrow 0} f^{(\ell)}(z)=0(\ell=0,1, \ldots),
$$

then the function $f$ vanishes identically on $R\left(c_{n}, r_{n}\right)$. It is an interesting but difficult problem to find a complete condition even for admissible sequences $(N(n))_{n \geq 1}$ to give the validity of the uniqueness theorem for $H^{\infty}(R(N(n)))$ at $z=0$. However the following partial result will be sufficient for our present purpose.

Proposition 3.1. If the uniqueness theorem is valid for $H^{\infty}(R(N(n)))$ at $z=0$, then $(N(n))_{n \geq 1}$ increases so rapidly as to satisfy the condition $(2.8): \lim _{n \rightarrow \infty} N(n)$ $=\infty$.

Unfortunately the converse of this is not true as will be shown later in 8.1 by the example $(n / \alpha)_{n \geq 1}$ with $\alpha \geq 2$. Two sufficient conditions will be given in Propositions 5.1 and 6.1 below. The proof of the above proposition will be given in $3.2-3.4$.

3.2. Proof of Proposition 3.1. We only have to show that the uniqueness theorem is invalid for $H^{\infty}(R)(R=R(N(n)))$ at $z=0$ if the condition (2.8) does not hold. Thus we assume that $\liminf _{n \rightarrow \infty} N(n)<\infty$, which implies the existence of a positive constant $\mu$ such that $\{n \in \mathbb{N}: N(n) \leq \mu\}$ is an infinite set. Fix an arbitrary sequence $\left(\mu_{n}\right)_{n \geq 1}$ of positive numbers $\mu_{n}$ satisfying

$$
\mu_{n} \geq \max (\mu, 2), \lim _{n \rightarrow \infty} \mu_{n}=\lim _{n \rightarrow \infty}\left(n-\mu_{n}\right)=\infty
$$

(e.g. $\mu_{n}=\mu+2+n / 2$ ). Then we can find an increasing sequence $\left(\nu_{n}\right)_{n \geq 1}$ of positive integers $\nu_{n}$ such that

$$
N\left(\nu_{n}\right) \leq \mu \quad(n \in \mathbb{N})
$$


and

$$
\alpha_{n}:=\left(n-\mu_{n}\right)-\frac{1}{\nu_{n}} \sum_{k=1}^{n-1} \nu_{k} \rightarrow \infty \quad(n \in \mathbb{N} ; \text { as } n \rightarrow \infty)
$$

(e.g. $\quad \nu_{n} \geq \frac{2}{n-\mu_{n}} \sum_{k=1}^{n-1} \nu_{k}$ when $n$ is large). Using these sequences $\left(\mu_{n}\right)_{n \geq 1}$ and $\left(\nu_{n}\right)_{n \geq 1}$ we define a new sequence $\left(c_{n}\right)_{n \geq 1}$ of centers $c_{n}$ and also a new sequence $\left(r_{n}\right)_{n \geq 1}$ of radii $r_{n}$ by

$$
c_{n}:=2^{-\nu_{n}}, r_{n}:=2^{-\nu_{n} \mu_{n}} \quad(n \in \mathbb{N}) .
$$

In view of $r_{n} \leq 2^{-\nu_{n} N\left(\nu_{n}\right)}(n \in \mathbb{N})$ by $(3.2)$, we see that

$$
S:=R\left(c_{n}, r_{n}\right) \supset R:=R\left(2^{-n}, 2^{-n N(n)}\right)=R(N(n)) .
$$

Hence, in order to show the invalidity of the uniqueness theorem for $H^{\infty}(R)$, it suffices to show that the uniqueness theorem for $H^{\infty}(S)$ is invalid. For this purpose we only have to construct a nonconstant $f$ in $H^{\infty}(S)$ satisfying (3.1). Consider the infinite product

$$
f(z):=\prod_{n \in \mathbb{N}}\left(1+\frac{c_{n}}{z-c_{n}}\right) \quad(z \in S),
$$

which will be seen in the sequel to be a required function. We consider auxiliary functions

$$
f_{p m}(z):=\prod_{p<n \leq m}\left(1+\frac{c_{n}}{z-c_{n}}\right) \quad(z \in S)
$$

for $p+1, m \in \mathbb{N}(p<m)$, and set

$$
f_{p}(z):=\lim _{m \rightarrow \infty} f_{p m}(z) .
$$

In particular $f_{0}(z)=f(z)$.

For simplicity we set $q_{n}(z):=\frac{c_{n}}{z-c_{n}}(n \in \mathbb{N})$. Since

$$
\left|q_{n}(z)\right| \leq 2 c_{n} /|z| \quad\left(|z| \geq c_{p} ; n>p\right),
$$

we see that $f_{p}(z)$ defines a meromorphic function on $0<|z| \leq \infty$ with $\left\{c_{n}: n>p\right\}$ as its pole set. Hence $f_{p}$ is holomorphic on $(\bar{S} \backslash\{0\}) \cup\left(\cup_{1 \leq n \leq p} \overline{\Delta_{n}}\right)$ and a fortiori on $\bar{S} \backslash\{0\}$, where we have set $\Delta_{n}:=\Delta\left(c_{n}, r_{n}\right)(n \in \mathbb{N})$ only in this proof.

3.3. We wish to show that $f_{p} \in H^{\infty}(S)$, i.e., $\sup _{z \in S}\left|f_{p}(z)\right|<\infty$, for each $p \in \mathbb{N}$. For this purpose we first show that

$$
\sup _{z \in \partial \Delta_{k}}\left|f_{p m}(z)\right| \leq A_{p}
$$

for $p<k \leq m$, where $A_{p}$ is a constant depending only on $p$. Since we have chosen as $\mu_{k} \geq 2$, it follows that

$$
\varepsilon_{k}:=2^{\nu_{k}} r_{k}=2^{-\nu_{k}\left(\mu_{k}-1\right)}<2^{-1}(k \in \mathbb{N}) .
$$


Firstly we see that there is a positive constant $B$ such that

$$
B_{p k}:=\prod_{p<n<k} \frac{1}{1-\left(1+\varepsilon_{k}\right) 2^{-\left(\nu_{k}-\nu_{n}\right)}} \leq B \quad(p+1, k \in \mathbb{N}, p+1<k) .
$$

Clearly, we see that $\nu_{k}-\nu_{n} \geq k-n$. If $k>n$, then $\left(1+\varepsilon_{k}\right) 2^{-\left(\nu_{k}-\nu_{n}\right)}<(3 / 2)$. $2^{-(k-n)}<1$. Noting that $(1-x)^{-1}$ is increasing for $x<1$, we have

$$
\begin{aligned}
B_{p k} & \leq \prod_{p<n<k} \frac{1}{1-(3 / 2) 2^{-(k-n)}}=\prod_{1 \leq j \leq k-p-1} \frac{1}{1-(3 / 2) 2^{-j}} \leq \prod_{j \geq 2} \frac{1}{1-3 \cdot 2^{-j}} \\
& =\prod_{j \geq 2}\left(1+\frac{3 \cdot 2^{-j}}{1-3 \cdot 2^{-j}}\right)=: B \quad(p+1, k \in \mathbb{N}, p+1<k) .
\end{aligned}
$$

Similarly there is a positive constant $C$ such that

$$
C_{k m}:=\prod_{k<n \leq m} \frac{1-\varepsilon_{k}}{1-\varepsilon_{k}-2^{-\left(\nu_{n}-\nu_{k}\right)}} \leq C \quad(k, m \in \mathbb{N}, k<m) .
$$

Fixing $k \in \mathbb{N}$, we next evaluate

$$
P_{n}:=\max _{z \in \partial \Delta_{k}}\left|1+q_{n}(z)\right|
$$

for three cases separately: $1 \leq n<k, n=k, n>k$.

The case $1 \leq n<k$ : we have

$$
\begin{aligned}
P_{n} & =\max _{z \in \partial \Delta_{k}}\left|\frac{z}{z-2^{-\nu_{n}}}\right| \leq \frac{2^{-\nu_{k}}+r_{k}}{2^{-\nu_{n}}-2^{-\nu_{k}}-r_{k}}=\frac{\left(1+2^{\nu_{k}} r_{k}\right) 2^{-\nu_{k}}}{2^{-\nu_{n}}-\left(1+2^{\nu_{k}} r_{k}\right) 2^{-\nu_{k}}} \\
& =\frac{\left(1+\varepsilon_{k}\right) 2^{-\left(\nu_{k}-\nu_{n}\right)}}{1-\left(1+\varepsilon_{k}\right) 2^{-\left(\nu_{k}-\nu_{n}\right)}} \leq \frac{2 \cdot 2^{-\left(\nu_{k}-\nu_{n}\right)}}{1-\left(1+\varepsilon_{k}\right) 2^{-\left(\nu_{k}-\nu_{n}\right)}} .
\end{aligned}
$$

The case $n=k$ : we have

$$
P_{n}=\max _{z \in \partial \Delta_{n}}\left|\frac{z}{z-2^{-\nu_{n}}}\right|=\frac{2^{-\nu_{k}}+r_{k}}{r_{k}}=\frac{1+\varepsilon_{k}}{2^{\nu_{k}} r_{k}} \leq 2 \cdot 2^{\nu_{k}\left(\mu_{k}-1\right)} .
$$

The case $n>k$ : we have

$$
\begin{aligned}
P_{n} & =\max _{z \in \partial \Delta_{k}}\left|1+q_{n}(z)\right| \leq 1+\max _{z \in \partial \Delta_{k}}\left|q_{n}(z)\right|=1+\max _{z \in \partial \Delta_{k}}\left|\frac{2^{-\nu_{n}}}{z-2^{-\nu_{n}}}\right| \\
& =1+\frac{2^{-\nu_{n}}}{\left(2^{-\nu_{k}}-r_{k}\right)-2^{-\nu_{n}}}=\frac{1-\varepsilon_{k}}{1-\varepsilon_{k}-2^{-\left(\nu_{n}-\nu_{k}\right)}} .
\end{aligned}
$$

In view of (3.3) we deduce that for $p<k \leq m$

$$
\begin{aligned}
M_{k} & :=\max _{z \in \partial \Delta_{k}}\left|f_{p m}(z)\right| \leq \prod_{p<n \leq m} P_{n}=\left(\prod_{p<n<k} P_{n}\right) \cdot P_{k} \cdot\left(\prod_{k<n \leq m} P_{n}\right) \\
& \leq\left(2^{k-p-1} 2^{-\sum_{p<n<k}\left(\nu_{k}-\nu_{n}\right)} B_{p k}\right) \cdot\left(2 \cdot 2^{\nu_{k}\left(\mu_{k}-1\right)}\right) \cdot C_{k m} \\
& =2^{k-p_{2}} 2^{-\left(k-p-\mu_{k}\right) \nu_{k}+\sum_{p<n<k} \nu_{n}} \cdot B_{p k} C_{k m} \\
& \leq 2^{\nu_{k}} 2^{-\left(\alpha_{k}-p\right) \nu_{k}} \cdot B_{p k} C_{k m} \leq B C 2^{-\left(\alpha_{k}-p-1\right) \nu_{k}} .
\end{aligned}
$$


From our choice of the sequence $\nu_{k}$ and the definition of $\alpha_{k}$ in (3.3), it follows that the value

$$
A_{p}=B C \max _{k>p} 2^{-\left(\alpha_{k}-p-1\right) \nu_{k}}
$$

is finite for each $p=0,1, \ldots$, and we conclude (3.4).

Note that the rational function $f_{p m}(z)$ is holomorphic on $\hat{\mathbb{C}} \backslash\left\{c_{p+1}, \ldots, c_{m}\right\}$, where $\hat{\mathbb{C}}:|z| \leq \infty$. The maximum principle yields $\left|f_{p m}(z)\right| \leq A_{p}(z \in S)$. Since $f_{p}=\lim _{m \rightarrow \infty} f_{p m}$, we conclude $f_{p} \in H^{\infty}(S)$.

3.4. It only remains to show that

$$
\lim _{z<0, z \rightarrow 0} f^{(\ell)}(z)=0 \quad(\ell=0,1, \ldots) .
$$

First we show that the domain $S=R\left(c_{n}, r_{n}\right)$ satisfies the condition (2.2). Noting $c_{n}=2^{-\nu_{n}}, r_{n}=2^{-\nu_{n} \mu_{n}}$, we have

$$
\begin{aligned}
\sum_{n=1}^{\infty} r_{n}\left(c_{n}-r_{n}\right)^{-(\ell+1)} & \leq \sum_{n=1}^{\infty} r_{n}\left(c_{n} / 2\right)^{-(\ell+1)}=\sum_{n=1}^{\infty} 2^{-\nu_{n} \mu_{n}+\left(\nu_{n}+1\right)(\ell+1)} \\
& =\sum_{n=1}^{\infty} 2^{-\nu_{n}\left(\mu_{n}-\ell-1-(\ell+1) / \nu_{n}\right)}
\end{aligned}
$$

Since $\nu_{n} \geq n$ and $\mu_{n} \rightarrow \infty$ by the way $\left(\mu_{n}\right)_{n \geq 1}$ was chosen, the final sum converges for $\ell=0,1, \ldots$. We can now apply the results obtained in 2.2-2.3 to the functions $f_{p}(z)$ in $H^{\infty}(S)$. Using (2.4), (2.5) and (2.6), we have

$$
\ell ! F_{p \ell}(0)=f_{p}^{(\ell)}(0)=\lim _{z<0, z \rightarrow 0} f_{p}^{(\ell)}(z) \quad(p, \ell=0,1, \ldots),
$$

where

$$
F_{p \ell}(z):=\frac{1}{2 \pi i} \int_{\Gamma} \frac{f_{p}(\zeta)}{\zeta^{\ell}(\zeta-z)} d \zeta \quad(z \in S, \Gamma:=\partial S \backslash\{0\} ; p, \ell=0,1, \ldots)
$$

We now prove $F_{0 \ell}(0)=f^{(\ell)}(0)=0$ by induction on $\ell$. Note that

$$
f(z)=f_{1}(z) \frac{z}{z-c_{1}} .
$$

Since $f_{1}(0)=\lim _{z<0, z \rightarrow 0} f_{1}(z)$ exists by $(3.5)$, we have $F_{00}(0)=f(0)=f_{1}(0) \cdot 0=0$. This proves the case $\ell=0$. Suppose that $F_{0 \ell}(0)=f^{(\ell)}(0)=0$ for $0 \leq \ell<k$ already have been proved. Then, we have $f(z)=z^{k} F_{0 k}(z)$ by $(2.7)$. Note that

$$
F_{0 k}(z)=f_{0, k+1}(z) f_{k+1}(z) / z^{k}=f_{k+1}(z) \cdot z \prod_{1 \leq n \leq k+1} \frac{1}{z-c_{n}}
$$

Applying (3.5) to the function $f_{k+1}$ with $\ell=0, f_{k+1}(0)=\lim _{z<0, z \rightarrow 0} f_{k+1}(z)$ exists. Thus $F_{0 k}(0)=f_{k+1}(0) \cdot 0=0$. We conclude $f^{(k)}(0)=F_{0 k}(0)=0$ as desired. 


\section{The MAIN THEOREM}

4.1. It is likely to happen that the Myrberg phenomenon occured for $(\tilde{R}, R, \varphi)$ $(R=R(N(n)))$ if $r_{n}=2^{-n N(n)}$ decreases enough rapidly as $n \rightarrow \infty$ since the Myrberg phenomenon as its prototype occurs for the extreme case $\left(\tilde{\Delta}_{0}, \Delta_{0}, \varphi\right)$ when each $r_{n}$ reduces to $r_{n}=0(n \in \mathbb{N})$. The same is true of the uniqueness theorem for $H^{\infty}(R)(R=R((N(n)))$. We now show as the main theorem of this paper that the rapidity of the decrease to make the latter valid is sufficient for the former to occur.

Theorem 4.1. If the uniqueness theorem is valid for $H^{\infty}(R)(R=R(N(n))$ at $z=0$, then the Myrberg phenomenon occurs for the covering surface $(\tilde{R}, R, \varphi)$.

4.2. Proof of Theorem 4.1. Take an arbitrary $g$ in $H^{\infty}(\tilde{R})$ with which an $f$ in $H^{\infty}(R)$ is determined by the following relation:

$$
f(z)=\left(g\left(z_{+}\right)-g\left(z_{-}\right)\right)^{2}
$$

for every $z$ in $R$, where $\varphi^{-1}(z)=\left\{z_{+}, z_{-}\right\}$. We have to show that $f$ is identically zero on $R$. Without loss of generality we may assume that $|g| \leq 1 / 2$ on $\tilde{R}$ so that $|f| \leq 1$ on $R$. By Proposition 3.1 we have

$$
\lim _{n \rightarrow \infty} N(n)=\infty
$$

For sufficiently large positive integers $n$ we consider annuli

$$
A_{n}=\left\{z: 2^{-n N(n)}<\left|z-2^{-n}\right|<2^{-n-2}\right\},
$$

which are contained in $R$. We choose a coordinate $w$ on the annulus $\varphi^{-1}\left(A_{n}\right)$ so as to satisfy the relation $\varphi(w)=w^{2}+2^{-n}$. Then $\varphi^{-1}\left(A_{n}\right)$ is represented by $w$ as

$$
\varphi^{-1}\left(A_{n}\right)=\left\{w: 2^{-n N(n) / 2}<|w|<2^{-n / 2-1}\right\}
$$

and $w=\varphi^{-1}(z)$ as $w=\sqrt{z-2^{-n}}$.

Take the concentric circle $\gamma_{n}:|w|=\left(2^{-n N(n) / 2} \cdot 2^{-n / 2-1}\right)^{1 / 2}$ in the annulus $\varphi^{-1}\left(A_{n}\right)$. We will estimate the diameter $\operatorname{diam}\left(g\left(\gamma_{n}\right)\right)$ of the image curve $g\left(\gamma_{n}\right)$ of $\gamma_{n}$ under the mapping $g$. For simplicity we set $2^{-n N(n) / 2}=a$ and $2^{-n / 2-1}=b$. Then $\varphi^{-1}\left(A_{n}\right)$ is $\{a<|w|<b\}$ and $\gamma_{n}:|w|=\sqrt{a b}$. Taking $w$ in $\gamma_{n}$ we use the Cauchy integral formula to deduce

$$
\begin{aligned}
\left|g^{\prime}(w)\right| & =\left|\frac{1}{2 \pi i} \int_{|\omega|=b} \frac{g(\omega)}{(\omega-w)^{2}} d \omega-\frac{1}{2 \pi i} \int_{|\omega|=a} \frac{g(\omega)}{(\omega-w)^{2}} d \omega\right| \\
& \leq \frac{1}{2 \pi}\left(\int_{|\omega|=b} \frac{1 / 2}{(b-\sqrt{a b})^{2}}|d \omega|+\int_{|\omega|=a} \frac{1 / 2}{(\sqrt{a b}-a)^{2}}|d \omega|\right) \\
& =\frac{1}{(\sqrt{b}-\sqrt{a})^{2}} .
\end{aligned}
$$


Using this estimate we see that

$$
\begin{aligned}
\operatorname{diam}\left(g\left(\gamma_{n}\right)\right) & \leq \frac{1}{2} \int_{\gamma_{n}}\left|g^{\prime}(w)\right||d w| \leq \frac{\pi \sqrt{a b}}{(\sqrt{b}-\sqrt{a})^{2}} \\
& =\pi \frac{\sqrt{a / b}}{(1-\sqrt{a / b})^{2}}=\pi \frac{\left(2^{-n N(n) / 2} 2^{n / 2+1}\right)^{1 / 2}}{\left(1-\left(2^{-n N(n) / 2} 2^{n / 2+1}\right)^{1 / 2}\right)^{2}} .
\end{aligned}
$$

Therefore we obtain the desired estimates

$$
\operatorname{diam}\left(g\left(\gamma_{n}\right)\right) \leq 2^{-n N(n) / 8}
$$

for all sufficiently large $n$. We set

$$
\rho_{n}:=2^{-n}+2^{-n N(n) / 2} 2^{-n / 2-1} .
$$

The point $\rho_{n}$ is in $A_{n}$ for every sufficiently large $n$ and $\left(\rho_{n}\right)_{+}$and $\left(\rho_{n}\right)_{-}$belong to $\gamma_{n}$, where we have set $\varphi^{-1}\left(\rho_{n}\right)=\left\{\left(\rho_{n}\right)_{+},\left(\rho_{n}\right)_{-}\right\}$. Hence we have

$$
\left|f\left(\rho_{n}\right)\right|=\left|g\left(\left(\rho_{n}\right)_{+}\right)-g\left(\left(\rho_{n}\right)_{-}\right)\right|^{2} \leq\left(\operatorname{diam}\left(g\left(\gamma_{n}\right)\right)\right)^{2} \leq 2^{-n N(n) / 4} .
$$

Since $\rho_{n}^{\ell}>2^{-\ell n}$ for every $\ell=0,1, \ldots$, we see by the above estimate that $\left|f\left(\rho_{n}\right) / \rho_{n}^{\ell}\right|$ $\leq 2^{-n(N(n) / 4-\ell)}$ and we conclude that

$$
\lim _{m \rightarrow \infty} f\left(\rho_{m}\right) / \rho_{m}^{\ell}=0 \quad(\ell=0,1, \ldots) .
$$

We now maintain that the following limits exist (cf. (2.5)):

$$
\lim _{m \rightarrow \infty} F_{\ell}\left(\rho_{m}\right)=F_{\ell}(0)=\frac{f^{(\ell)}(0)}{\ell !} \quad(\ell=0,1, \ldots) .
$$

For this purpose we estimate the absolute value of the integrand in the integral defining $F_{\ell}\left(\rho_{m}\right)$ on every integrating domain $\partial \Delta_{n}$ separately for sufficiently large m. First for $n=m$

$$
\begin{aligned}
\max _{\zeta \in \partial \Delta_{n}}\left|\frac{f(\zeta)}{\zeta^{\ell}\left(\zeta-\rho_{n}\right)}\right| & \leq \frac{1}{\left(2^{-n}-2^{-n N(n)}\right)^{\ell}\left(2^{-n N(n) / 2} 2^{-n / 2-1}-2^{-n N(n)}\right)} \\
& \leq 2 /\left(2^{-\ell n} \cdot 2^{-n N(n) / 2} 2^{-n / 2-1}\right) \leq 2^{2 n N(n) / 3}
\end{aligned}
$$

since $n=m$ is sufficiently large. Next for sufficiently large $n$ with $n \neq m$ we have

$$
\max _{\zeta \in \partial \Delta_{n}}\left|\frac{f(\zeta)}{\zeta^{\ell}\left(\zeta-\rho_{m}\right)}\right| \leq \frac{1}{\left(2^{-n}-2^{-n N(n)}\right)^{\ell} \cdot 2^{-n N(n) / 2}} \leq 2^{2 n N(n) / 3} .
$$

Thus the absolute value of the integrand in the integral defining $F_{\ell}\left(\rho_{m}\right)$ is dominated by an integrable step function independent of $m$. Therefore the Lebesgue dominated convergence theorem allows us to take the limit under the integral sign to conclude the required relation (4.4).

We finally prove inductively that $f(z)=z^{\ell} F_{\ell}(z)$ and $f^{(\ell)}(0)=0$ for every $\ell=0,1, \ldots$ (cf. Lemma 2.1). Observe that $F_{0}(z)=f(z)$, and (4.3) and (4.4) for $\ell=0$ assure that $f^{(0)}(0)=0$. By $(2.7)$ for $\ell=1$ we see that $f(z)=z^{1} F_{1}(z)$. By assuming $f(z)=z^{\ell} F_{\ell}(z)$ and $f^{(k)}(0)=0$ for $0 \leq k \leq \ell,(2.7)$ for $\ell+1$ again yields

$$
f(z)-\frac{f^{(\ell+1)}(0)}{(\ell+1) !} z^{\ell+1}=z^{\ell+2} F_{\ell+2}(z) .
$$


This implies that

$$
\frac{f^{(\ell+1)}(0)}{(\ell+1) !}=\frac{f\left(\rho_{m}\right)}{\rho_{m}^{\ell+1}}-\rho_{m} F_{\ell+2}\left(\rho_{m}\right) .
$$

By (4.3) for $\ell+1$ and (4.4) for $\ell+2$ we see on making $m \rightarrow \infty$ in the above identity that $\frac{f^{(\ell+1)}(0)}{(\ell+1) !}=0-0 \cdot F_{\ell+2}(0)=0$. By the mathematical induction we can conclude that $f^{(\ell)}(0)=0(\ell=0,1, \ldots)$. Therefore the validity of the uniqueness theorem for $H^{\infty}(R)$ at 0 yields that $f \equiv 0$ on $R$.

\section{IRREGULAR BOUNDARY POINT}

5.1. We suppose that the radii $2^{-n N(n)}$ of removed discs $\Delta_{n}$ of $R=R(N(n))$ decrease so rapidly as to satisfy

$$
\sum_{n=1}^{\infty} 1 / N(n)<\infty
$$

which of course implies $N(n) \rightarrow \infty(n \rightarrow \infty)$. The condition is equivalent to

$$
\sum_{n=1}^{\infty} n / \log \left(1 / 2^{-n N(n)}\right)<\infty
$$

which is nothing but the Wiener criterion (cf. e.g. p.104 in [15] or (5.1.15') in [9]): (5.2) is necessary and sufficient for the point $z=0$ to be an irregular boundary point of the region $R$ in the sense of potential theory. Thus assuming (5.1) amounts to the same to assuming that the point $z=0$ is irregular for the region $R$. We have the following result (cf. [11]).

Proposition 5.1. If the condition (5.1) is satisfied for $R=R(N(n))$ (i.e. if the origin 0 is irregular for the region $R$ ), then the uniqueness theorem is valid for $H^{\infty}(R)$ at 0.

5.2. Proof of Proposition 5.1. Choose an arbitrary $f$ in $H^{\infty}(R)$ with $\lim _{z<0, z \rightarrow 0}$ $f^{(\ell)}(z)=0$ for every $\ell=0,1, \ldots$. We have to show that $f \equiv 0$ on $R$. Contrariwise we assume that $f \not \equiv 0$ on $R$. Without loss of generality we may suppose that $|f|<1$ on $R$. Thus $\log (1 /|f(z)|)$ is a positive superharmonic function on $R$.

We take another sequence of closed discs $\Delta_{n}^{\prime}:=\bar{\Delta}\left(2^{-n},\left(2^{-n N(n)}\right)^{1 / 2}\right)(n=$ $1,2, \ldots)$. These discs are mutually disjoint for sufficiently large $n$. By changing the radii suitably, if necessary, for the first finite number of discs $\Delta_{n}^{\prime}$ under the restrictions $\Delta_{n}^{\prime} \supset \Delta_{n}$, we may assume that $\Delta_{n}^{\prime}$ are all contained in $\Delta_{0}$ and mutually disjoint. Consider the region

$$
S:=\Delta_{0} \backslash \bigcup_{n=1}^{\infty} \Delta_{n}^{\prime}
$$

We maintain that there exists a positive harmonic function $h(z)$ on $S$ with boundary values zero on $\partial S \backslash\{0\}$ such that

$$
0<h(z)<\log (1 /|z|) \quad(z \in S) .
$$

Observe that the condition (5.2) is equivalent to $\sum_{n=1}^{\infty} n / \log \left(1 /\left(2^{-n N(n)}\right)^{1 / 2}\right)<\infty$ so that the point $z=0$ is also an irregular boundary point for the smaller region 
$S$ in the sense of the Dirichlet problem. Let $G(z, \zeta)$ be the Green function for the region $S$. The Bouligand theorem says that

$$
\limsup _{\zeta \in S, \zeta \rightarrow 0} G(z, \zeta)>0
$$

for one and hence for every $z$ in $R$. By the Harnack inequality, there exists a sequence $\left\{\zeta_{n}\right\}$ in $S$ converging to 0 such that $\left(G\left(z, \zeta_{n}\right)\right)_{n \geq 1}$ converges to a positive harmonic function $h(z)$ locally uniformly on $S$. Since each $G\left(z, \zeta_{n}\right)$ has vanishing boundary values on $\partial S \backslash\{0\}$ and each component $\partial \Delta_{n}^{\prime}$ of $\partial S \backslash\{0\}$ is a circle, considering an annular subdomain of $S$ with $\partial \Delta_{n}^{\prime}$ as its inside boundary circle, an application of the maximum principle for harmonic functions shows that the limit function $h(z)$ must have boundary values zero on $\partial S \backslash\{0\}$. Moreover, by the comparison principle, we have

$$
G\left(z, \zeta_{n}\right)<\log \left|\frac{1-\bar{\zeta}_{n} z}{z-\zeta_{n}}\right| \quad\left(z \in S \backslash\left\{\zeta_{n}\right\}\right) .
$$

On letting $n \rightarrow \infty$, we obtain (5.3). Since we have (2.8), by Lemma 2.1 the condition $\lim _{z<0, z \rightarrow 0} f^{(\ell)}(z)=0(\ell=0,1, \ldots)$ is equivalent to $f(z)=z^{\ell} F_{\ell}(z)(z \in R)$ for every $\ell=1,2, \ldots$ By $(2.8)$ we see that

$$
C_{\ell}:=\sup _{z \in S}\left|F_{\ell}(z)\right| \leq \sum_{n=0}^{\infty} \frac{1}{2 \pi} \int_{\partial \Delta_{n}} \frac{1}{|\zeta|^{\ell}\left(2^{-n N(n) / 2}-2^{-n N(n)}\right)}|d \zeta|<\infty
$$

for every $\ell=0,1, \ldots$. Hence, we have $|f(z)| \leq C_{\ell}|z|^{\ell}$ for $z$ in $\bar{S} \backslash\{0\}$, or equivalently, $\log (1 /|f(z)|)$ dominates the function $\ell \log (1 /|z|)-\log C_{\ell}$ for every $\ell=0,1, \ldots$. Hence in particular by (5.3) we obtain

$$
\log \frac{1}{|f(z)|} \geq \ell h(z)-\log C_{\ell}(z \in S \backslash\{0\} ; \ell=0,1, \ldots) .
$$

Fix an arbitrary positive number $\varepsilon$ and consider the superharmonic function

$$
s(z)=s_{\ell, \varepsilon}(z):=\log \frac{1}{|f(z)|}+\varepsilon \log \frac{1}{|z|}-\ell h(z)
$$

on $\bar{S} \backslash\{0\}$. Clearly $s \geq 0$ on $\partial S \backslash\{0\}$. By (5.4) and by the presence of the term $\varepsilon \log \frac{1}{|z|}$, we have $\lim _{z \in S, z \rightarrow 0} s(z)=+\infty$. Therefore we can conclude that

$$
\liminf _{z \in S, z \rightarrow \zeta} s(z) \geq 0(\zeta \in \partial S) .
$$

By the minimum principle for superharmonic functions we see that $s(z)=s_{\ell, \varepsilon}(z) \geq$ $0(z \in S)$. On letting $\varepsilon$ tend to zero we now conclude that

$$
\log \frac{1}{|f(z)|} \geq \ell h(z)(\ell=0,1, \ldots)
$$

for any $z$ in $S$, which is a desired contradiction. 
5.3. In view of the main theorem 4.1 and Proposition 5.1 we obtain the following result (Theorem A, cf. [5], [6]).

Theorem 5.1. If the origin 0 is an irregular boundary point of $R=R(N(n))$ in the sense of potential theory, then the Myrberg phenomenon occurs for the surface $(\tilde{R}, R, \varphi)$.

\section{Quasi analytic Character}

6.1. With an admissible sequence $(N(n))_{n \geq 1}$ we associate a sequence $(M(\ell))_{\ell \geq 1}$ in $\mathbb{N} \cup\{\infty\}$ determined by the following relation

$$
M(\ell):=\min \{m \in \mathbb{N}: N(\nu) \geq \ell+2(\nu \geq m)\}
$$

under the convention $\min \emptyset=+\infty$. Clearly $(M(\ell))_{\ell \geq 1}$ is a nondecreasing sequence divergent to $\infty ;(M(\ell))_{\ell \geq 1} \subset \mathbb{N}$ if and only if $\lim _{n \rightarrow \infty} N(n)=\infty$. Roughly speaking the more $(N(n))_{n \geq 1}$ diverges to $\infty$ rapidly, the more $(M(\ell))_{\ell \geq 1}$ diverges to $\infty$ slowly, and vice versa. In terms of the associated sequence $(M(\ell))_{\ell \geq 1}$ we consider the following condition for the sequence $(N(n))_{n \geq 1}$ :

$$
\sum_{\ell=1}^{\infty} 1 / 2^{M(\ell)}=\infty
$$

This condition clearly assures that $(M(\ell))_{\ell \geq 1} \subset \mathbb{N}$ so that $(N(n))_{n \geq 1}$ satisfies (2.8). We will later see that this condition (6.2) has nothing to do with the condition (5.1), i.e. the boundary point $z=0$ of the region $R=R(N(n))$ may or may not be irregular in the sense of potential theory. We maintain the following result (cf. [12]).

Proposition 6.1. If $(N(n))_{n \geq 1}$ diverges to $\infty$ so rapidly as to satisfy the condition (6.2), then the uniqueness theorem is valid for $H^{\infty}(R)(R=R(N(n))$ at $z=0$.

The proof will be given in 6.3 below. From the above result combined with the main theorem 4.1 we immediately derive the following result.

Theorem 6.1. If the sequence $(N(n))_{n \geq 1}$ satisfies (6.2), then the Myrberg phenomenon occurs for the covering surface $(\tilde{R}, R, \varphi)$.

6.2. Before proceeding to the proof of Proposition 6.1 we insert here the following celebrated classical result which will be essentially made use of below in the next Subsection 6.3. Assume that a holomorphic function $g(z)$ defined on the disc $W=$ $\Delta(-1 / 4,1 / 4)=\{z:|z+1 / 4|<1 / 4\}$ touching the origin $z=0$ satisfies the inequalities:

$$
|g(z)| \leq C \tau_{\ell}|z|^{\ell} \quad(z \in W ; \ell=0,1, \ldots),
$$

where $C$ is a positive constant independent of $\ell$ and $\left(\tau_{\ell}\right)_{\ell \geq 1}$ is a sequence of positive numbers. Then the following result holds. 
Lemma 6.1 (The Carleman uniqueness theorem). If the sequence $\left(\tau_{\ell}\right)_{\ell \geq 1}$ satisfies the condition

$$
\sum_{\ell=1}^{\infty}\left(\tau_{\ell}\right)^{-1 / \ell}=\infty
$$

then the function $g(z)$ vanishes identically on the disc $W$.

This is a part and actually a trivial variant of the Ostrowski theorem [13], which is partly a generalization and partly a reformulation of the original Carleman Theorem [1]. We recommend for the reader unfamiliar with the proof of the above lemma to read $\S 1$ in pp. 194-205 of the above Ostrowski old paper [13], which presents a clear and easily readable reasoning.

6.3. Proof of Proposition 6.1. Take an arbitrary $f \in H^{\infty}(R)$ with $f^{(\ell)}(0)=0$ $(\ell=0,1, \ldots)$. We are to show that $f \equiv 0$ on $R$. We first estimate $\left|F_{\ell}(z)\right|$ given by (2.5) for $f$ when $z$ varies in the disc $W=\Delta(-1 / 4,1 / 4)$ which is contained in $R$ and whose periphery contains the origin 0 . The result is :

$$
\sup _{z \in W}\left|F_{\ell}(z)\right| \leq \sigma^{\ell} \sum_{n=0}^{\infty} 2^{n(\ell+1-N(n))} \quad(\ell=1,2, \ldots),
$$

where $\sigma=\sup _{\ell \in \mathbb{N}} 2^{(1+1 / \ell)}\|f\|_{\infty}^{1 / \ell}\left(\|f\|_{\infty}=\sup _{R}|f|\right)$ is a finite constant. Here we understand that the term corresponding to $n=0$ is 1 in the summation of the right hand side of (6.5). To show (6.5) observe that

$$
\left|F_{\ell}(z)\right| \leq \sum_{n=0}^{\infty} \frac{1}{2 \pi} \int_{\Gamma_{n}} \frac{\|f\|_{\infty}}{|\zeta|^{\ell}|\zeta-z|}|d \zeta|
$$

for $z$ in $W$. First for $n=0$, since $|\zeta|=1$ and $|\zeta-z| \geq 1 / 2$ for $z \in W$ and $\zeta \in \Gamma_{0}$, we have

$$
\frac{1}{2 \pi} \int_{\Gamma_{0}} \frac{\|f\|_{\infty}}{|\zeta|^{\ell}|\zeta-z|}|d \zeta| \leq 2\|f\|_{\infty}<\sigma^{\ell} .
$$

Next, for $n \geq 1$, since $|\zeta|,|\zeta-z| \geq 2^{-(n+1)}$ for $z \in W$ and $\zeta \in \Gamma_{n}$, we have

$$
\begin{aligned}
\frac{1}{2 \pi} \int_{\Gamma_{n}} \frac{\|f\|_{\infty}}{|\zeta|^{\ell}|\zeta-z|}|d \zeta| & \leq\left(2^{n+1}\right)^{\ell+1}\|f\|_{\infty} 2^{-n N(n)} \\
& \leq \sigma^{\ell} 2^{n(\ell+1-N(n))} .
\end{aligned}
$$

Hence we can conclude that (6.5) is valid.

From (6.5) we derive the inequalities

$$
|f(z)| \leq \sigma^{\ell} 2^{\ell M(\ell)}|z|^{\ell} \quad(z \in W ; \ell \geq 2) .
$$

Since $f(z)=z^{\ell} F_{\ell}(z)$, for the proof of (6.6), we only have to show that

$$
\sup _{z \in W}\left|F_{\ell}(z)\right| \leq \sigma^{\ell} 2^{\ell M(\ell)} \quad(z \in W ; \ell \geq 2) .
$$


But in view of $(6.5)$ it is sufficient to prove that

$$
\sum_{n=0}^{\infty} 2^{n(\ell+1-N(n))} \leq 2^{\ell M(\ell)} \quad(\ell \geq 2) .
$$

This is shown as follows. We view the left hand side of the above as the sum

$$
\sum_{0 \leq n<M(\ell)} 2^{n(\ell+1-N(n))}+\sum_{n \geq M(\ell)} 2^{n(\ell+1-N(n))} .
$$

By recalling the definition of $M(\ell)$ we see that $N(n) \geq 1$ trivially for all $n$ and hence for $0 \leq n<M(\ell)$ and that $N(n) \geq \ell+2$ for $n \geq M(\ell)$. Therefore the above is dominated by

$$
\sum_{0 \leq n<M(\ell)} 2^{\ell n}+\sum_{n \geq M(\ell)} 2^{-n}=\frac{2^{\ell M(\ell)}-1}{2^{\ell}-1}+2^{1-M(\ell)},
$$

which is at most $2^{\ell M(\ell)}$ if $\ell \geq 2$.

Now apply Lemma 6.1 to $g=f \mid W$. The condition (6.3) is valid for $C=$ $\max \left(1,\|f\|_{\infty}\right)$ and $\tau_{\ell}:=\sigma^{\ell} 2^{\ell M(\ell)}$. By (6.2) we see that

$$
\sum_{\ell=1}^{\infty}\left(\tau_{\ell}\right)^{-1 / \ell}=\sigma^{-1} \sum_{\ell=1}^{\infty} 1 / 2^{M(\ell)}=\infty
$$

i.e. (6.4) is satisfied. Therefore, by Lemma $6.1, f \mid W=0$ and hence $f$ vanishes identically on $R$.

\section{REgular BOUNDARY POINT}

7.1. We now come to the stage of being able to answer negatively, as one of the main purposes of this paper, to the question whether the irregularity of the point $z=0$ in Theorem A or Theorem 5.1 is also necessary for the validity of the Myrberg phenomenon for $(\tilde{R}, R, \varphi)$.

Theorem 7.1. There is an $R=R(N(n))$ such that $z=0$ is a regular boundary point of $R$ in the sense of potential theory and yet the Myrberg phenomenon is valid for the surface $(\tilde{R}, R, \varphi)$.

In view of Theorem 6.1 we only have to show the existence of an admissible sequence $(N(n))_{n \geq 1}$ satisfying (6.2) and not satisfying (5.1). We denote by $\mathcal{Q}(\mathcal{I}$, resp.) the family of admissible sequences $(N(n))_{n \geq 1}$ satisfying the condition (6.2) ( (5.1), resp.). We also denote by $\mathcal{N}$ the family of all admissible sequence $(N(n))_{n \geq 1}$ satisfying (2.8): $\lim _{n \rightarrow \infty} N(n)=\infty$. Clearly $\mathcal{Q} \cup \mathcal{I} \subset \mathcal{N}$. Then our main task is to show that $\mathcal{Q} \backslash \mathcal{I}$ is not empty, which is achieved below in Subsection 7.5. In passing we will also remark that $\mathcal{I} \backslash \mathcal{Q}, \mathcal{Q} \cap \mathcal{I}$, and $\mathcal{N} \backslash(\mathcal{Q} \cup \mathcal{I})$ are all not empty.

It may also be convenient to introduce the notation $\mathcal{U}(\mathcal{M}$, resp.) to mean the family of admissible sequence $(N(n))_{n \geq 1}$ such that the uniqueness theorem is valid for $H^{\infty}(R)$ at $z=0$ (the Myrberg phenomenon is valid for $(\tilde{R}, R, \varphi)$, resp.), where $R=R(N(n))$. Then what we have shown in this paper may be restated as the string of inclusion relations:

$$
\mathcal{Q} \cup \mathcal{I} \subset \mathcal{U} \subset \mathcal{M} \cap \mathcal{N} \subset \mathcal{M}
$$


An important open question is to determine whether the three inclusions in the above string are proper or not. This problem is completely left open for future study.

7.2. Proof of $\mathcal{I} \backslash \mathcal{Q} \neq \emptyset$. We maintain that $\left(n^{2}\right)_{n \geq 1} \in \mathcal{I} \backslash \mathcal{Q}$. Since $\sum_{n \geq 1} 1 / n^{2}<$ $\infty,\left(n^{2}\right)_{n \geq 1}$ certainly belongs to $\mathcal{I}$. By the definition of the associated sequence $(M(\ell))_{\ell \geq 1}$ of $\left(n^{2}\right)_{n \geq 1}$, we have

$$
M(\ell)=[\sqrt{\ell+2}]^{*} \quad(\ell \in \mathbb{N}),
$$

where $[\cdot]^{*}$ is the modified Gaussian symbol, i.e. $[\xi]^{*}$ is the smallest integer not less than the real number $\xi$. Since $M(\ell)>\sqrt{\ell}$, we have

$$
\begin{aligned}
\sum_{\ell \in \mathbb{N}} 1 / 2^{M(\ell)} & \leq \sum_{\ell \in \mathbb{N}} 1 / 2^{\sqrt{\ell}}=\sum_{k \in \mathbb{N}}\left(\sum_{k^{2} \leq \ell<(k+1)^{2}} 1 / 2^{\sqrt{\ell}}\right) \\
& \leq \sum_{k \in \mathbb{N}}(2 k+1) / 2^{k}<\infty
\end{aligned}
$$

and thus $\left(n^{2}\right)_{n \geq 1}$ does not belong to $\mathcal{Q}$ and therefore belongs to $\mathcal{I} \backslash \mathcal{Q}$, as desired.

7.3. Proof of $\mathcal{Q} \cap \mathcal{I} \neq \emptyset$. We claim that $\left(2^{n}\right)_{n \geq 1} \in \mathcal{Q} \cap \mathcal{I}$. Since $\sum_{n \geq 1} 1 / 2^{n}<\infty$, $\left(2^{n}\right)_{n \geq 1} \in \mathcal{I}$. By the definition of the associated sequence $(M(\ell))_{\ell \geq 1}$ of $\left(2^{n}\right)_{n \geq 1}$, we have

$$
M(\ell)=\left[\log _{2}(\ell+2)\right]^{*} \quad(\ell \in \mathbb{N}) .
$$

Since $M(\ell) \leq \log _{2} 2(\ell+2)$, we see that $1 / 2^{M(\ell)} \geq 1 / 2(\ell+2)$ and thus

$$
\sum_{\ell \in \mathbb{N}} 1 / 2^{M(\ell)} \geq \sum_{\ell \in \mathbb{N}} 1 / 2(\ell+2)=\infty
$$

or $\left(2^{n}\right)_{n \geq 1} \in \mathcal{Q}$. Therefore $\left(2^{n}\right)_{n \geq 1} \in \mathcal{Q} \cap \mathcal{I}$.

7.4. Proof of $\mathcal{N} \backslash(\mathcal{Q} \cup \mathcal{I}) \neq \emptyset$. We will see that $(n / \alpha)_{n \geq 1} \in \mathcal{N} \backslash(\mathcal{Q} \cup \mathcal{I})$ for every fixed $\alpha>0$. Since $(n / \alpha)_{n \geq 1} \rightarrow \infty(n \rightarrow \infty)$, we trivially have $(n / \alpha)_{n \geq 1} \in \mathcal{N}$. It is also clear that $(n / \alpha)_{n \geq 1} \notin \mathcal{I}$, i.e. $\sum_{n \geq 1} 1 /(n / \alpha)=\infty$. By the definition of the associated sequence $(M(\ell))_{\ell \geq 1}$ of $(n / \alpha)_{n \geq 1}$, we have

$$
M(\ell)=[(\ell+2) \alpha]^{*} \quad(\ell \in \mathbb{N}) .
$$

Since $M(\ell) \geq(\ell+2) \alpha$, we have

$$
\sum_{\ell \in \mathbb{N}} 1 / 2^{M(\ell)} \leq \sum_{\ell \in \mathbb{N}} 1 / 2^{(\ell+2) \alpha}=2^{-2 \alpha} \sum_{\ell \in \mathbb{N}}\left(1 / 2^{\alpha}\right)^{\ell}<\infty
$$

or $(N(n))_{n \geq 1}$ does not belong to $\mathcal{Q}$. Therefore we conclude that $(n / \alpha)_{n \geq 1} \in \mathcal{N} \backslash$ $(\mathcal{Q} \cup \mathcal{I})$. 
7.5. Proof of $\mathcal{Q} \backslash \mathcal{I} \neq \emptyset$ (cf. [12]). We will construct a sequence belonging to $\mathcal{Q} \backslash \mathcal{I}$. First we consider an auxiliary sequence $\left(\nu_{m}\right)_{m \geq 0}$ in $\mathbb{N} \cup\{0\}$ given inductively as follows:

$$
\nu_{m+1}=\nu_{m}+2^{\nu_{m}} \quad(m=0,1, \ldots), \nu_{0}=0 .
$$

Therefore the sequence $\left(\nu_{m}\right)_{m \geq 0}$ increases considerably rapidly; the first few terms are $\left(\nu_{m}\right)_{m \geq 0}=(0,1,3,11,2059, \ldots)$. The sequence $\left(\nu_{m}\right)_{m \geq 0}$ is used to divide $\mathbb{N}$ into infinite blocks $\left(\mathbb{N}_{m}\right)_{m \geq 1}$ as follows: $\mathbb{N}=\cup_{m \in \mathbb{N}} \mathbb{N}_{m}$ with

$$
\mathbb{N}_{m}=\left\{n \in \mathbb{N}: \nu_{m-1}+1 \leq n<\nu_{m}\right\} \quad(m \in \mathbb{N}) .
$$

The first few blocks of $\left(\mathbb{N}_{m}\right)_{m \geq 1}$ are

$$
\{\underline{1}\},\{2, \underline{3}\},\{4,5,6,7,8,9,10, \underline{11}\},\{12,13, \ldots, 2058, \underline{2059}\}, \ldots
$$

Observe that the last term in each block forms the sequence $\left(\nu_{m}\right)_{m \geq 1}$. The sequence $(N(n))_{n \geq 1}$ is now given by the following:

$$
N(n)=\nu_{m}+2 \quad\left(n \in \mathbb{N}_{m} ; m \in \mathbb{N}\right) .
$$

Thus $N(n)$ is a constant $\nu_{m}+2$ on each block $\mathbb{N}_{m}$ and therefore the sequence $(N(n))_{n \geq 1}$ looks like

$$
(N(n))_{n \geq 1}=(3,5,5,13,13,13,13,13,13,13,13,2061, \ldots, 2061, \ldots) .
$$

We need to know the associated sequence $(M(\ell))_{\ell \geq 1}$ with the present sequence $(N(n))_{n \geq 1}$ given by (7.2). By the definition of $M(\ell)$ we instantly see that

$$
M\left(\nu_{m}+\nu\right)=\nu_{m}+1 \quad\left(1 \leq \nu \leq 2^{\nu_{m}}\right)
$$

for every $m=0,1, \ldots$ so that $(M(\ell))_{\ell \geq 1}$ looks like

$$
(M(\ell))_{\ell \geq 1}=(1,2,2,4,4,4,4,4,4,4,4,12, \ldots, 12, \ldots) .
$$

We now see that $(N(n))_{n \geq 1} \in \mathcal{Q}$. In fact,

$$
\begin{aligned}
\sum_{\ell \in \mathbb{N}} 1 / 2^{M(\ell)} & =\sum_{0 \leq m<\infty}\left(\sum_{1 \leq \nu \leq 2^{\nu_{m}}} 1 / 2^{M\left(\nu_{m}+\nu\right)}\right) \\
& \geq \sum_{0 \leq m<\infty}\left(1 / 2^{\nu_{m}+1}\right) \cdot 2^{\nu_{m}}=\sum_{0 \leq m<\infty} 1 / 2=\infty .
\end{aligned}
$$

We complete the proof by showing that $(N(n))_{n \geq 1} \notin \mathcal{I}$. Since $N\left(\nu_{m}+\nu\right)=$ $\nu_{m+1}+2=\nu_{m}+2^{\nu_{m}}+2\left(1 \leq \nu \leq 2^{\nu_{m}}\right)$ and since $\nu 2^{-\nu} \rightarrow 0$ as $\nu \rightarrow \infty$, the evaluation goes as follows:

$$
\begin{aligned}
\sum_{n \in \mathbb{N}} 1 / N(n) & =\sum_{0 \leq m<\infty}\left(\sum_{1 \leq \nu \leq 2^{\nu_{m}}} 1 / N\left(\nu_{m}+\nu\right)\right)=\sum_{0 \leq m<\infty} 2^{\nu_{m}} /\left(\nu_{m}+2^{\nu_{m}}+2\right) \\
& =\sum_{0 \leq m<\infty} 1 /\left(1+\left(\nu_{m}+2\right) 2^{-\nu_{m}}\right)=\infty
\end{aligned}
$$

Hence $(N(n))_{n \geq 1}$ given by $(7.2)$ belongs to $\mathcal{Q} \backslash \mathcal{I}$. 


\section{INVALIDITy OF THE MYRBERG PHENOMENON}

8.1. Thus far we have been concerned with the positive direction on the validity of the Myrberg phenomenon. In this last section we turn to the negative direction. Recall ([7]) that the Myrberg phenomenon is valid for the surface $(\tilde{R}, R, \varphi)(R=$ $R(N(n)))$ if and only if there is a point $a \in R$ such that $f\left(a_{+}\right)=f\left(a_{-}\right)$for every $f \in H^{\infty}(\tilde{R})$, where $\varphi^{-1}(a)=\left\{a_{+}, a_{-}\right\}$. Thus to maintain the invalidity of the Myrberg phenomenon for $(\tilde{R}, R, \varphi)$ we only have to exhibit an $f \in H^{\infty}(\tilde{R})$ with $f\left(a_{+}\right) \neq f\left(a_{-}\right)$for one and hence sufficiently more than needed for every $a \in R$. For this purpose we consider the situation that the sequence $(N(n))_{n \geq 1}$ is slowly increasing in the sense that

$$
\sup _{n \in \mathbb{N}} n\left(N(n)-\frac{n+1}{2}\right)<\infty .
$$

A typical example of such a sequence is $(n / \alpha)_{n \geq 1}$ with $\alpha \geq 2$. Then we have the following result.

Theorem 8.1. If the condition (8.1) is satisfied, then the Myrberg phenomenon is invalid for the surface $(\tilde{R}, R, \varphi)(R=R(N(n)))$.

The proof of this theorem will be given in Subsection 8.3 below. In view of the main theorem 4.1 we can immediately deduce from the above the following result.

Corollary 8.1. If the condition (8.1) is satisfied, then the uniqueness theorem is invalid for $H^{\infty}(R)(R=R(N(n)))$ at $z=0$.

We have seen in $\S 3$ that $\mathcal{U} \subset \mathcal{N}$ in the notation of $\S 7$. By the above result we see that $(n / \alpha)_{n \geq 1} \in \mathcal{N} \backslash \mathcal{U}$ with $\alpha \geq 2$, i.e. the condition (2.8) is necessary but not sufficient for the validity of the uniqueness theorem for $H^{\infty}(R(N(n)))$ at $z=0$.

8.2. Consider the infinite product $p(z)$ for each $z$ in $\hat{\mathbb{C}} \backslash\{0\}$ (where $\hat{\mathbb{C}}:|z| \leq \infty)$ given by

$$
p(z):=\prod_{n \in \mathbb{N}}\left(1+\frac{2^{-n}}{z-2^{-n}}\right)=\prod_{n \in \mathbb{N}} \frac{z}{z-2^{-n}} .
$$

Since $\left|2^{-m} /\left(z-2^{-m}\right)\right| \leq 2^{-(m-1)} /|z|\left(|z| \geq 2^{-(n-1)} ; m \geq n\right)$ for every $n \in \mathbb{N}$, $p(z)$ converges almost uniformly on $\hat{\mathbb{C}} \backslash\{0\}$ and hence $p(z)$ is certainly a meromorphic function on $\hat{\mathbb{C}} \backslash\{0\}$ with $\left\{2^{-n}: n \in \mathbb{N}\right\}$ as its pole set. In particular, $p(z)$ is holomorphic on $\bar{R} \backslash\{0\}(R=R(N(n)))$. The meaning of the condition (8.1) is clarified by the following assertion.

Proposition 8.1. The function $p(z)$ in (8.2) belongs to $H^{\infty}(R)(R=R(N(n)))$ if and only if the condition (8.1) is satisfied.

The proof of this result will be given below in Subsections 8.4-8.6. 
8.3. Proof of Theorem 8.1. Since (8.1) is assumed, Proposition 8.1 yields $p \in$ $H^{\infty}(R)$. Regarding the function $p(z)$ as the product of functions $z^{2} /\left(z-2^{-2 n+1}\right)(z-$ $\left.2^{-2 n}\right)(n \in \mathbb{N})$, we see that the square root $f:=\sqrt{p}$ defines a single valued holomorphic function on $\tilde{R}$ so that $f \in H^{\infty}(\tilde{R})$. For any $a \in R$, let $\varphi^{-1}(a)=\left\{a_{+}, a_{-}\right\}$. Since $f^{2}-p=0$, we have $f\left(a_{+}\right) f\left(a_{-}\right)=-p(a) \neq 0$ and $f\left(a_{+}\right)+f\left(a_{-}\right)=0$ so that $f\left(a_{+}\right) \neq f\left(a_{-}\right)$, i.e. $f$ separates $\varphi^{-1}(a)$ for every $a \in R$.

8.4. Proof of Proposition 8.1. The proof is divided into three steps. The first step given in this subsection 8.4, a general observation on the boundedness of $p$ on $R$ is made. The necessity of (8.1) is given as the second step in Subsection 8.5. In the final subsections 8.6-8.7, the sufficiency of (8.1) is given as the third step. For simplicity we write $r_{n}:=2^{-n N(n)}$ and $q_{n}(z):=2^{-n} /\left(z-2^{-n}\right)(n \in \mathbb{N})$ in this proof. Then the condition (8.1) takes the following form:

$$
\inf _{n \in \mathbb{N}} 2^{\frac{n(n+1)}{2}} \cdot r_{n}>0
$$

Recall that $p(z)$ is holomorphic not only on $R$ but also on $\bar{R} \backslash\{0\}$. We can thus consider

$$
M_{n}:=\max _{z \in \partial \Delta_{n}}|p(z)|(n \in \mathbb{N})
$$

which constitute a sequence $\left(M_{n}\right)_{n \geq 1}$ of finite positive numbers. Let $\gamma_{n}$ be the circle $|z|=2^{-n}-r_{n}$. In view of $\gamma_{n} \cap \partial \Delta_{n}=\left\{2^{-n}-r_{n}\right\}$ (one point set) and

$$
\left|1+q_{m}(z)\right|=\frac{|z|}{\left|z-2^{-m}\right|}=\frac{2^{-n}-r_{n}}{\left|z-2^{-m}\right|}\left(z \in \gamma_{n}\right)
$$

for each $m \in \mathbb{N}$, we see that

$$
\begin{aligned}
\max _{z \in \gamma_{n}}\left|1+q_{m}(z)\right| & =\left(2^{-n}-r_{n}\right) / \min _{z \in \gamma_{n}}\left|z-2^{-m}\right| \\
& =\left(2^{-n}-r_{n}\right) /\left|2^{-n}-r_{n}-2^{-m}\right|=\left|1+q_{m}\left(2^{-n}-r_{n}\right)\right| .
\end{aligned}
$$

Hence we have

$$
\max _{z \in \gamma_{n}}|p(z)|=\left|p\left(2^{-n}-r_{n}\right)\right| \leq M_{n}(n \in \mathbb{N})
$$

Set $R_{n}:=R \cap\left\{|z|>2^{-n}-r_{n}\right\}$ and $R_{n}^{\prime}:=\left\{|z|>2^{-n}-r_{n}\right\} \backslash \bigcup_{1 \leq k \leq n} \Delta_{k}$. By the maximum modulus principle for holomorphic functions, we see that

$$
\sup _{z \in R_{n}}|p(z)|=\sup _{z \in R_{n}^{\prime}}|p(z)|=\max \left\{M_{1}, \ldots, M_{n}\right\}(n \in \mathbb{N}) .
$$

From this it follows that

$$
\sup _{z \in R}|p(z)|=\sup _{n \in \mathbb{N}} M_{n}
$$

since $p$ is holomorphic in $\hat{\mathbb{C}} \backslash\{0\} \cup\left\{2^{-n}: n \in \mathbb{N}\right\}$ and hence $|p|$ does not take its supremum over $R$ on $\Gamma_{0}$. 
8.5. We show, owing a lot to Professor Akio Osada, the necessity of the condition (8.1), i.e. $\inf _{n \in \mathbb{N}} 2^{n(n+1) / 2} \cdot r_{n}>0$, for $p(z)$ to be bounded on $R$. We consider the sequence $\left(A_{n}\right)_{n \geq 1}$ given by $A_{1}:=1$ and

$$
A_{n}:=\prod_{1 \leq j<n}\left(1+\frac{2^{-j}}{1-2^{-j}}\right)(n=2,3, \ldots) .
$$

The sequence $\left(A_{n}\right)_{n \geq 1}$ is increasingly convergent to the limit

$$
A:=\lim _{n \rightarrow \infty} A_{n}=\prod_{j \in \mathbb{N}}\left(1+\frac{2^{-j}}{1-2^{-j}}\right) \in(1, \infty) .
$$

In terms of these quantities we obtain

$$
A \cdot A_{n} \cdot\left(2^{\frac{n(n+1)}{2}} \cdot r_{n}\right)^{-1} \leq M_{n}(n \in \mathbb{N})
$$

To show this, observe that

$$
\log |p(z)|=\sum_{m \in \mathbb{N} \backslash\{n\}} \log \left|\frac{z}{z-2^{-m}}\right|+\log |z|-\log r_{n}
$$

for $z=2^{-n}+r_{n} e^{i \theta} \in \partial \Delta_{n}$. From (8.3) it follows that

$$
\frac{1}{2 \pi} \int_{\partial \Delta_{n}} \log |p(z)| d \theta \leq \log M_{n}
$$

Applying the Gauss mean value theorem to the left hand side of the above we obtain

$$
\sum_{m \in \mathbb{N} \backslash\{n\}} \log \left|\frac{2^{-n}}{2^{-n}-2^{-m}}\right|+\log 2^{-n}-\log r_{n} \leq \log M_{n},
$$

or equivalently

$$
\left(\prod_{m<n} \frac{2^{-n}}{2^{-m}-2^{-n}}\right) \cdot\left(\prod_{m>n} \frac{2^{-n}}{2^{-n}-2^{-m}}\right) \cdot \frac{2^{-n}}{r_{n}} \leq M_{n} .
$$

The first factor of the left hand side of the above equals

$$
\begin{aligned}
\prod_{m<n} \frac{2^{-(n-m)}}{1-2^{-(n-m)}} & =\prod_{1 \leq j<n} \frac{2^{-j}}{1-2^{-j}}=2^{-(1+2+\cdots+(n-1))} \prod_{1 \leq j<n} \frac{1}{1-2^{-j}} \\
& =2^{-n(n-1) / 2} \prod_{1 \leq j<n}\left(1+\frac{2^{-j}}{1-2^{-j}}\right)=2^{-n(n-1) / 2} A_{n} .
\end{aligned}
$$

The second factor is equal to

$$
\prod_{m>n} \frac{1}{1-2^{-(m-n)}}=\prod_{j \geq 1} \frac{1}{1-2^{-j}}=\prod_{j \geq 1}\left(1+\frac{2^{-j}}{1-2^{-j}}\right)=A .
$$

Summing up these observations, we deduce (8.5). 
Note that $A A_{n}>A_{n}^{2}>A_{1}^{2}>1$. Hence (8.5) and (8.4) imply

$$
2^{n(n+1) / 2} \cdot r_{n} \geq A A_{n} / M_{n}>1 / \sup _{R}|p|>0
$$

which assures the validity of (8.1).

8.6. Finally we prove the sufficiency of the condition of (8.1), i.e. $\inf _{n \in \mathbb{N}} 2^{n(n+1) / 2}$. $r_{n}>0$, for $p(z)$ to be bounded on $R$. Put $\varepsilon_{n}:=2^{n} r_{n}(n \in \mathbb{N})$. Since $2^{-(n+1)} \notin \Delta_{n}$, we see that $\varepsilon_{n}<1 / 2$. We next show that

$$
M_{n} \leq\left(1+\varepsilon_{n}\right)^{n} \cdot B_{n} C_{n} \cdot\left(2^{n(n+1) / 2} \cdot r_{n}\right)^{-1}(n \in \mathbb{N}),
$$

where

$$
B_{n}:=\prod_{1 \leq j<n}\left(1+\frac{\left(1+\varepsilon_{n}\right) 2^{-j}}{1-\left(1+\varepsilon_{n}\right) 2^{-j}}\right), \quad C_{n}:=\prod_{j \in \mathbb{N}}\left(1+\frac{2^{-j}}{1-\varepsilon_{n}-2^{-j}}\right) .
$$

Obviously, the two sequences $\left(B_{n}\right)_{n \geq 1}$ and $\left(C_{n}\right)_{n \geq 1}$ are bounded.

To prove (8.6) we only have to estimate each term of $|p(z)|=\prod_{m \geq 1}\left|1+q_{m}(z)\right|$ from above on $\partial \Delta_{n}$. For this purpose, fixing an $n$, we evaluate

$$
Q_{m}:=\max _{z \in \partial \Delta_{n}}\left|1+q_{m}(z)\right|
$$

separately for three cases: $m<n, m=n$, and $m>n$.

The case $m<n: \Delta_{n}$ is situated on the left hand side of $2^{-m}$ and thus the minimal distance from points in $\partial \Delta_{n}$ to $2^{-m}$ is $2^{-m}-\left(2^{-n}+r_{n}\right)$ and the maximal distance from points in $\partial \Delta_{n}$ to the origin $z=0$ is $2^{-n}+r_{n}$. Hence we have

$$
\begin{aligned}
Q_{m} & =\max _{z \in \partial \Delta_{n}}\left|\frac{z}{z-2^{-m}}\right| \leq \frac{2^{-n}+r_{n}}{2^{-m}-2^{-n}-r_{n}} \\
& =\frac{\left(1+2^{n} r_{n}\right) 2^{-n}}{2^{-m}-\left(1+2^{n} r_{n}\right) 2^{-n}}=\frac{\left(1+\varepsilon_{n}\right) 2^{-(n-m)}}{1-\left(1+\varepsilon_{n}\right) 2^{-(n-m)}} .
\end{aligned}
$$

The case $m=n$ : This time the distance from points in $\partial \Delta_{n}$ to $2^{-n}$ is the constant $r_{n}$ and the maximal distance from points in $\partial \Delta_{n}$ to the origin 0 is $2^{-n}+r_{n}$. Thus, as above, we have

$$
Q_{n}=\max _{z \in \partial \Delta_{n}}\left|\frac{z}{z-2^{-n}}\right|=\frac{2^{-n}+r_{n}}{r_{n}}=\frac{\left(1+2^{n} r_{n}\right) 2^{-n}}{r_{n}}=\frac{1+\varepsilon_{n}}{2^{n} r_{n}} .
$$

The case $m>n$ : Since $\Delta_{n}$ is situated on the right hand side of $2^{-m}$, the minimal distance from points in $\partial \Delta_{n}$ to $2^{-m}$ is $\left(2^{-n}-r_{n}\right)-2^{-m}$. Hence, on viewing $\left|1+q_{m}(z)\right| \leq 1+\left|q_{m}(z)\right|$, we have

$$
\begin{aligned}
Q_{m} & \leq 1+\max _{z \in \partial \Delta_{n}}\left|\frac{2^{-m}}{z-2^{-m}}\right| \leq 1+\frac{2^{-m}}{\left(2^{-n}-r_{n}\right)-2^{-m}} \\
& =1+\frac{2^{-m}}{\left(1-2^{n} r_{n}\right) 2^{-n}-2^{-m}}=1+\frac{2^{-(m-n)}}{\left(1-\varepsilon_{n}\right)-2^{-(m-n)}} .
\end{aligned}
$$


In view of

$$
M_{n}=\max _{z \in \partial \Delta_{n}}|p(z)| \leq \prod_{m \in \mathbb{N}} Q_{m}=\left(\prod_{m<n} Q_{m}\right) \cdot Q_{n} \cdot\left(\prod_{m>n} Q_{m}\right)
$$

we estimate each of the three factors on the rightmost term of the above separately. First we have

$$
\begin{aligned}
\prod_{m<n} Q_{m} & \leq \prod_{m<n} \frac{\left(1+\varepsilon_{n}\right) 2^{-(n-m)}}{1-\left(1+\varepsilon_{n}\right) 2^{-(n-m)}}=\prod_{1 \leq j<n} \frac{\left(1+\varepsilon_{n}\right) 2^{-j}}{1-\left(1+\varepsilon_{n}\right) 2^{-j}} \\
& =\left(1+\varepsilon_{n}\right)^{n-1} \cdot 2^{-(1+2+\cdots+(n-1))} \prod_{1 \leq j<n} \frac{1}{1-\left(1+\varepsilon_{n}\right) 2^{-j}} \\
& =\left(1+\varepsilon_{n}\right)^{n-1} \cdot 2^{-n(n-1) / 2} \prod_{1 \leq j<n}\left(1+\frac{\left(1+\varepsilon_{n}\right) 2^{-j}}{1-\left(1+\varepsilon_{n}\right) 2^{-j}}\right) \\
& =\left(1+\varepsilon_{n}\right)^{n-1} \cdot 2^{-n(n-1) / 2} \cdot B_{n},
\end{aligned}
$$

which together with the estimate of $Q_{n}$ implies

$$
\left(\prod_{m<n} Q_{m}\right) \cdot Q_{n} \leq\left(1+\varepsilon_{n}\right)^{n} \cdot\left(2^{n(n+1) / 2} \cdot r_{n}\right)^{-1} \cdot B_{n}
$$

Finally we have

$$
\prod_{m>n} Q_{m} \leq \prod_{m>n}\left(1+\frac{2^{-(m-n)}}{\left(1-\varepsilon_{n}\right)-2^{-(m-n)}}\right)=\prod_{j \in \mathbb{N}}\left(1+\frac{2^{-j}}{1-\varepsilon_{n}-2^{-j}}\right)=C_{n} .
$$

Thus the inequality (8.6) has been established.

8.7. Let us look at the right hand side of (8.6). By virtue of $\varepsilon_{n}<1 / 2,\left(B_{n}\right)_{n \geq 1}$ and $\left(C_{n}\right)_{n \geq 1}$ are bounded sequences. Since we are assuming (8.1), the last factor also form a bounded sequence $\left(\left(2^{n(n+1) / 2} \cdot r_{n}\right)^{-1}\right)_{n \geq 1}$. Unfortunately the sequence $\left(\left(1+\varepsilon_{n}\right)^{n}\right)_{n \geq 1}$ may not be bounded. For example, since the choice $r_{n}=2^{-n} / 4$ $(n \in \mathbb{N})$ gives an admissible $(N(n))_{n \geq 1}$ satisfying (8.1), it may happen that $\varepsilon_{n}=1 / 4$ so that $\left(\left(1+\varepsilon_{n}\right)^{n}\right)_{n \geq 1}=\left(5^{n} / 4^{n}\right)_{n \geq 1}$ is not bounded. To eliminate this undesirable situation, set $\delta:=\inf _{n \in \mathbb{N}} 2^{n(n+1) / 2} r_{n}>0$. Then

$$
r_{n}^{\prime}:=2^{-n(n+1) / 2} \cdot \delta \leq r_{n}(n \in \mathbb{N}) .
$$

By using $\bar{\Delta}\left(2^{-n}, r_{n}^{\prime}\right)$ in place of $\Delta_{n}=\bar{\Delta}\left(2^{-n}, r_{n}\right)(n \in \mathbb{N})$, we replace $R$ by $S:=$ $\Delta_{0} \backslash \cup_{n \in \mathbb{N}} \bar{\Delta}\left(2^{-n}, r_{n}^{\prime}\right)$. Since $S \supset R$, the boundedness of $p(z)$ on $S$ implies that of $p(z)$ on $R$. For this reason we may assume from the beginning that

$$
r_{n}=2^{-n(n+1) / 2} \cdot \delta(\delta>0 ; n \in \mathbb{N})
$$

in oder to prove the boundedness of $p(z)$. Then, for sufficiently large $n$,

$$
\varepsilon_{n}=2^{n} r_{n}=2^{-n(n-1) / 2} \cdot \delta \leq 1 / n
$$

and therefore

$$
\left(1+\varepsilon_{n}\right)^{n} \leq(1+1 / n)^{n}<e .
$$


This finishes the proof of the boundedness of the sequence $\left(M_{n}\right)_{n \geq 1}$ and a fortiori that of the boundedness of $p(z)$ on $R$.

\section{REFERENCES}

1. T. Carlemann, Les Fonction Quasi Analytiqies, Gauthier-Villars, 1926.

2. S. Fisher, Function Theory on Planar Domains, John Wiley \& Sons, 1983.

3. M. Hayashi, Smootheness of analytic functions at boundary points, Pacific J. Math., 67 (1976), $171-202$.

4. M. Hayashi and T. Kato, Point separation of a two-sheeted disc by bounded analytic functions, Hokkaido Math. J. (to appear).

5. M. Hayashi and M. Nakai, Point separation by bounded analytic functions of a covering Riemann surface, Pacific J. Math., 134 (1988), 261-273.

6. - On the Myrberg type phenomenon, Analytic Function Theory of One Complex Variable (ed. by Y. Komatsu, K. Niino and C. C. Yang, eds.), Pitman Research Notes in Math. Sci., 212(1989), pp.1-12.

7. M. Hayashi, M. Nakai and S. Segawa, Bounded analytic functions on two sheeted discs, Trans. Amer. Math. Soc., 333 (1992), 799-819.

8. $293-325$.

9. N. S. Landkof, Foundations of Modern Potential Theory, Springer, 1972.

10. P. J. Myrberg, Über die Analytische Fortsetzung von beschränkten Funktionen, Ann. Acad. Sci. Fenn., Ser. A, 58 (1949), 1-7.

11. M. Nakai, Valuations on meromorphic functions of bounded type, Trans. Amer. Math. Soc., 309 (1988), 231-252.

12. $161-167$.

13. A. Ostrowski, Über quasianalytische Funktionen und Bestimmtheit asymptotischer Entwickelungen, Acta Math., 53 (1929), 181-266.

14. L. Sario and M. Nakai, Classification Theory of Riemann Surfaces, Springer, 1970.

15. M. Tsuji, Potential Theory in Modern Function Theory, Chelsea, 1975.

16. L. Zalcman, Bounded analytic functions on domains of infinite connectivity, Trans. Amer. Math. Soc., 144 (1969), 241-269.

(Mikiniro Hayashi) Department of Mathematics, Hokkaido University, Sapporo 0600810, JAPAN

E-mail address: hayashi@math.sci.hokudai.ac.jp

(Mitsuru Nakai) Department of Mathematics, Nagoya Institute of Technology, Gokiso, Showa, Nagoya 466-0061, JAPAN (Professor Emeritus); Mailing address: 52 Eguchi, HinAGA, CHITA 478-0041, JAPAN

E-mail address: nakai@daido-it.ac.jp 